\title{
Magnetic resonance imaging for deep infiltrating endometriosis: current concepts, imaging technique and key findings
}

\author{
Filomenamila Lorusso ${ }^{1}$, Marco Scioscia ${ }^{2}$, Dino Rubini ${ }^{1}$, Amato Antonio Stabile lanora', Doriana Scardigno ${ }^{3}$,
} Carla Leuci ${ }^{1}$, Michele De Ceglie ${ }^{1}$, Angela Sardaro ${ }^{4}$, Nicola Lucarelli ${ }^{1}$ and Arnaldo Scardapane ${ }^{1 *}$ (D)

\begin{abstract}
Endometriosis is an estrogen-dependent chronic disease affecting about 10\% of reproductive-age women with symptoms like pelvic pain and infertility. Pathologically, it is defined by the presence of endometrial tissue outside the uterine cavity responsible for a chronic inflammatory process. For decades the diagnosis of endometriosis was based on surgical exploration and biopsy of pelvic lesions. However, laparoscopy is not a risk-free procedure with possible false negative diagnosis due to an underestimate of retroperitoneal structures such as ureters and nerves. For these reasons nowadays, the diagnosis of endometriosis is based on a noninvasive approach where clinical history, response to therapy and imaging play a fundamental role. Trans-vaginal ultrasound and magnetic resonance imaging are suitable for recognizing most of endometriotic lesions; nevertheless, their accuracy is strictly determined by operators' experience and imaging technique. This review paper aims to make radiologists aware of the diagnostic possibilities of pelvic MRI and familial with the MR acquisition protocols and image interpretation for women with endometriosis.
\end{abstract}

Keywords: Endometriosis, Deep infiltrating endometriosis, Magnetic resonance imaging, Imaging protocol

\section{Key points}

- Diagnostic laparoscopy is considered the gold standard for endometriosis, but it is invasive with possible false negative results.

- Nowadays there is a paradigm shift from surgical to non-invasivediagnosis based on symptoms, response to therapy and imaging.

- MRI is highly accurate for the diagnosis of Deep Infiltrating Endometriosis.

- The diagnostic results of MRI depend on an accurate imaging technique and on the comprehension of specific MR-findings.

\footnotetext{
*Correspondence: arnaldo.scardapane@uniba.it; arnaldo.scardapane@gmail. com

'University of Bari Medical School - Interdisciplinary Department of Medicine, Section of Diagnostic Imaging, Piazza Giulio Cesare, 11, 70124 Bari, Italy

Full list of author information is available at the end of the article
}

\section{Background}

Endometriosis, particularly deep pelvic infiltrating endometriosis (DIE), is a clinical issue affecting premenopausal women who may experience severe pelvic pain and infertility [1]. These symptoms are mainly associated with the growth of endometrial tissue outside the uterine cavity, with consequent chronic inflammatory reactions and fibromuscular hyperplasia affecting the pelvic peritoneum and the pelvic wall and organs [2]. The disease affects approximately $10 \%$ of women of reproductive age and is diagnosed in approximately $20 \%-50 \%$ of infertile women and nearly $90 \%$ of infertile women with chronic pelvic pain [3]. Accurate early diagnosis of DIE is crucial to provide women with early tailored treatments and avoid inappropriate surgery [4]. Nevertheless, although many diagnostic techniques have been used, early diagnosis of DIE remains a major challenge [5].

Laparoscopic exploration is considered the diagnostic golden standard, because it allows for direct visualization 
of lesions; however, it is not a risk-free surgery and may underestimate retroperitoneal structures, such as nerves and ureters, with possible false negative procedures [6, 7]. Because a poor correlation has been demonstrated between the symptoms and severity of lesions, some authors suggest a paradigmatic shift to a more clinical diagnostic approach based on the combination of symptoms, imaging findings and response to empiric treatment, even before any surgical confirmation [8]. In this scenario, transvaginal ultrasound, magnetic resonance imaging (MRI) and in some cases computed tomography (CT) play a fundamental role $[9,10]$. MRI, the imaging technique with the highest overall accuracy for assessing the extent of DIE, has high specificity for endometriotic foci, owing to its inherent soft-tissue resolution [11, 12]. Nevertheless, to achieve the expected accuracy, the examination itself and the image interpretation should be tailored to each woman's specific issues. This review provides radiologists with information on how to obtain good quality MRI images, interpret and report them correctly.

\section{Management of deep endometriosis: current concepts} Endometriosis is a complex and heterogeneous disease that may manifest with three clinical patterns with increasing severity. Superficial peritoneal lesions are characterized by superficial implants of the pelvic peritoneum; ovarian endometriomas (OMA) are hemorrhagic cysts arising from ectopic endometrial tissue growing within the ovaries and less frequently outside the ovaries $[13,14]$; and DIE leads to the most severe clinical pattern and is characterized by ectopic endometrial tissue penetrating deeper than $5 \mathrm{~mm}$ under the peritoneal surface, thus leading to local inflammation and consequently to fibrosis and muscular hyperplasia $[7,15,16]$. DIE is usually found as a multifocal disease simultaneously involving multiple pelvic sites such as the Douglas pouch, the utero-sacral ligaments (USL), pelvic nerves, the rectum, the bladder, and the ureters $[17,18]$ (Table 1). The pathophysiology of such lesions is widely unknown; the type of lesion may vary over the life course, with no evidence supporting an ordered progression of endometriotic lesions [19]. Similarly, the heterogeneity of symptoms is high. Women with endometriosis may experience dysmenorrhea, dyspareunia, dysuria, constipation, chronic pelvic pain and infertility; however, a clear characterization of the pain types and topologies of implants is lacking [20]. Consequently, some women with minimal disease may report severe pelvic pain and infertility, whereas others with diffuse pelvic lesions can be almost asymptomatic [6]. The heterogeneity of the disease and the uncertainties about its pathogenesis make its diagnosis challenging. For decades, laparoscopic visualization
Table 1 Most frequent pelvic localization of DIE

\begin{tabular}{ll}
\hline Site & Frequency (\%) \\
\hline Pouch of Douglas/retrocervical & $55-60$ \\
Uterosacral ligaments & $32-57$ \\
Recto-vaginal septum & $20-48$ \\
Bowel (overall) & $16-35$ \\
Rectum & $30-40$ \\
Recto-sigmoid junction & $25-30$ \\
Sigmoid & $15-20$ \\
Cecum/appendix & 5 \\
Small bowel & 5 \\
Bladder & $5-8$ \\
Sacral nerves & $3-5$
\end{tabular}

References no. [12, 18, 37, 46, 53]

with histologic verification of lesions was considered the golden standard for diagnosis [21]. However, laparoscopy, even if diagnostic, is not a risk-free surgery. Furthermore, deep infiltrating endometriosis may not be clearly seen in some cases during diagnosis laparoscopy [22]. In fact, the diagnosis of adenomyosis and deep endometriosis involving retroperitoneal structures, particularly ureters and nerve roots, is extremely challenging, particularly when performed by non-experienced gynecologists. False negative procedures may result, thus significantly delaying the start of appropriate management and potentially leading to major complications $[7,8]$. Symptoms and clinical findings of endometriosis can result in clinical diagnoses that may be strongly supported by imaging techniques even without histological confirmation. Transvaginal ultrasound, because of its non-invasiveness, dynamicity, ease of use, availability, cost-effectiveness and reproducibility, is currently considered by many endometriosis experts as the best firstline method for assessment of DIE [4, 5, 23]. A systematic sonographic approach as defined by International Deep Endometriosis Analysis (IDEA) consensus was shown to improve detection rates of pelvic lesions [24]. MRI is also considered a highly accurate imaging modality in the evaluation of DIE, particularly when involvement of the rectum, ureters and nerve roots is suspected; these areas are highly important for both the patient and the surgeon and may be poorly visualized even with laparoscopy [25, 26]. MRI is also increasingly used to assess the anatomic response to medical or surgical treatment and to differentiate endometriosis from adenomyosis; the latter is a specific and heterogeneous disease contributing, independently of endometriosis to symptoms, defined as the invasion of endometrial tissue into the myometrium occurring in different forms (diffuse, focal, cystic or superficial). Adenomyosis may exist on its own but in about $30 \%$ of cases it is associated with DIE $[7,27]$. 
The shift toward clinical and imaging-based diagnosis shortens the time between the first consultation and the final diagnosis [28], but the use of non-invasive methods requires a rigorous approach to ensure meaningful and consistent results $[8,29]$.

\section{MRI acquisition protocol: dos, don'ts and maybes}

MRI is a widely used technique for the diagnosis of DIE; however, an international consensus on the best imaging protocol is lacking. Recent guidelines published by the European Society of Urogenital Radiology describe consensus suggestions from a conference among nine imaging centers in Europe and one in Japan [29]; however, the indications and imaging protocols may vary among institutions according to local expertise. In general, when suspicion of endometriosis exists, MRI should be used first to provide an adequate anatomic representation of

Table 2 Standard MRI protocol for endometriosis in our center

\begin{tabular}{llllll}
\hline Sequence & Plane & $\begin{array}{l}\text { Voxel } \mathbf{m m} \\
\text { (AP-RL- } \\
\text { thickness) }\end{array}$ & FOV $(\mathbf{m m})$ & NEX & TE \\
\hline TSET2 & $\begin{array}{c}\text { Axial/Obl } \\
\text { axial }\end{array}$ & $0.9-0.9-3$ & $280-350$ & 2 & 100 \\
TSET2 & Sagittal & $0.9-0.9-3$ & $180-250$ & 2 & 100 \\
TSET2 & Coronal & $0.8-0.8-3$ & $280-300$ & 2 & 100 \\
TSET1 & Axial & $0.9-0.9-3$ & $280-350$ & 1 & Shortest \\
THRIVE & Axial & $0.75-0.75-3$ & $280-350$ & 3 & Shortest \\
THRIVE & Sagittal & $0.75-0.75-3$ & $280-350$ & 3 & Shortest \\
Optional sequences & & & & \\
CE-THRIVE & Axial/Sagittal & $0.75-0.75-3$ & $280-350$ & 3 & Shortest \\
BTFE* & Axial/sagittal & $1.5-1.5-4$ & $280-350$ & 1 & Shortest \\
SSFSET2* & Axial/sagittal & $1-1-4$ & $280-350$ & 1 & 100 \\
\hline
\end{tabular}

*Performed with rectal distension the entire pelvis and its organs and second to ensure the recognition of DIE, according to the contrast between normal pelvic fatty tissue and endometriotic lesions or on the detection of hemorrhagic cysts and foci (Table 2).

\section{DOS}

MRI for endometriosis should be performed with a $1.5 \mathrm{~T}$ or $3 \mathrm{~T}$ scanner and high-resolution phased array coils (with 8-16 channels), whereas low-magnetic field or open-MRI lacks sufficient image quality to image DIE. High-resolution, thin section $(3 \mathrm{~mm})$ TSE-T2w sequences in the sagittal, axial and coronal planes are crucial to evaluate DIE, whereas TSE T1w (with and without fat saturation) should always be obtained to depict adnexal hemorrhagic lesions such as OMA. Oblique planes may be highly useful to visualize specific anatomical structures such as utero-sacral ligaments (Fig. 1) [30]. A substantial improvement in image quality may be obtained by using rectal cleansing and anti-peristaltic agents such as butyl-scopolamine or glucagon which can also be helpful in the evaluation of adenomyosis. Some authors suggest a more reliable effect of such agents when intravenous rather than intramuscular injection is used; however, intramuscular administration ensures longer anti-peristaltic results, in line with an average imaging duration of $20-25 \mathrm{~min}$ [31]. The pelvis should be imaged regardless of the phase of the menstrual cycle, in patients with a moderately full bladder [29].

\section{DON'Ts}

Because the recognition of DIE is based on the contrast between the high signal intensity of fatty tissue and low signal intensity of endometriotic nodules, fat-saturated T2w images should not be used [32, 33]. Among T1w fatsaturated techniques, STIR sequences should be avoided.

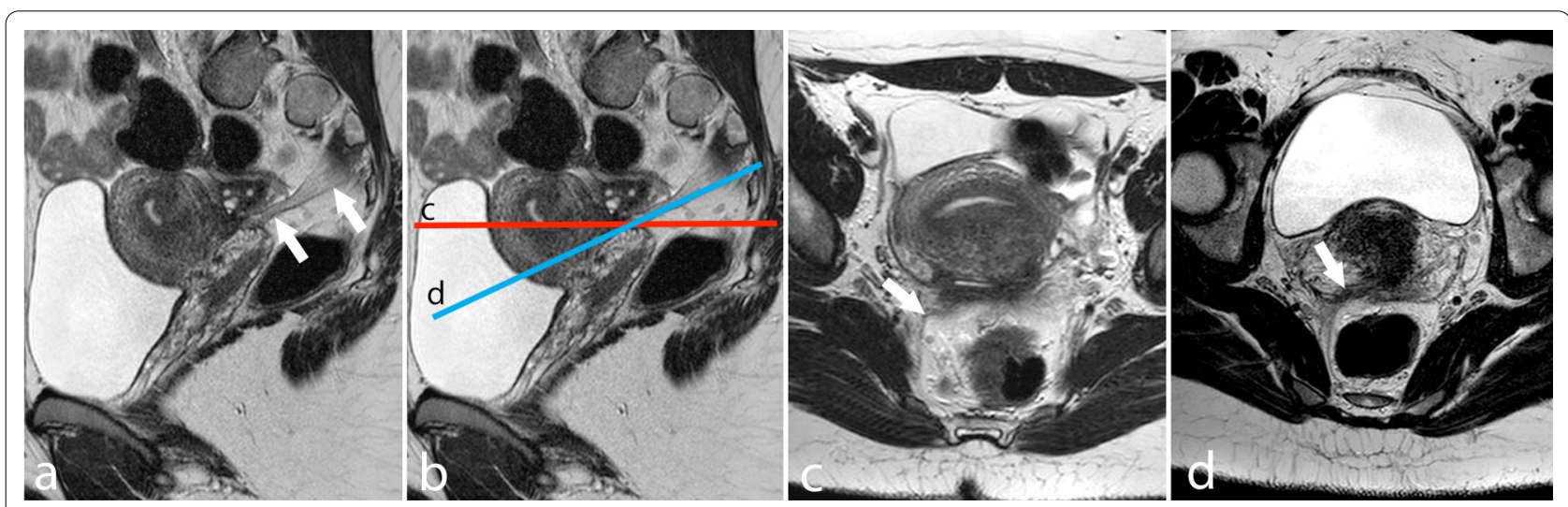

Fig. 1 Mild thickening of the right USL in a woman with DIE. a-b T2w sagittal images. cT2w axial image obtained along the red plane shown in $\mathbf{b}$ d T2w oblique axial image obtained along the blue plane shown in $\mathbf{b}$. The USL produces a better depiction of the sagittal and oblique axial plane (arrows) 


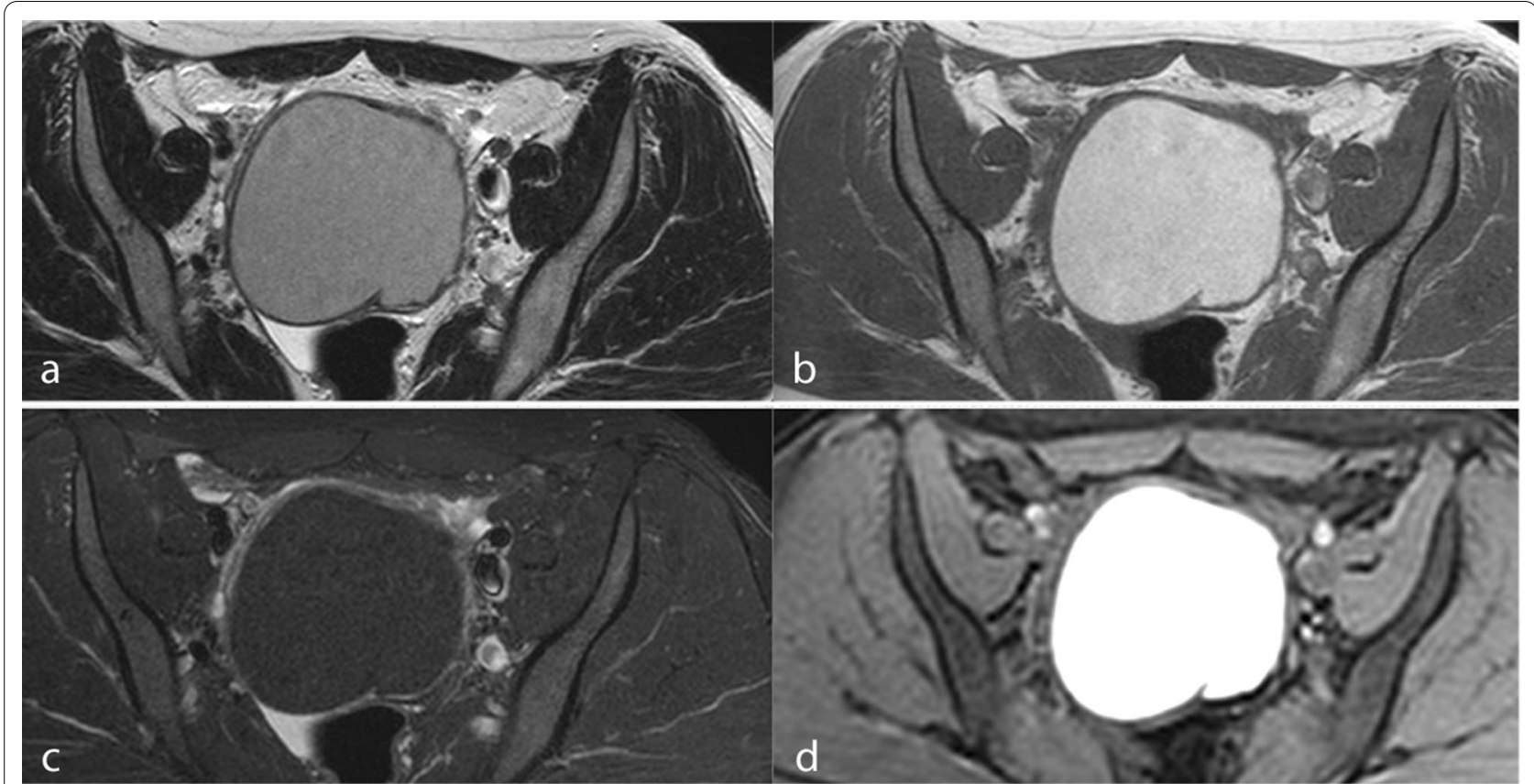

Fig. 2 OMA mimicking a mature cystic teratoma in a STIR sequence. (a) TSE T2w axial image, (b) TSE T1w axial image, (c) STIR axial image and (d) THRIVE axial image. The loss of T1w high signal intensity in the STIR image is not specific to fat (c), because endometriomas and fatty tissue may have similar T1 relaxation times. In the THRIVE sequence, on the basis of a spectral saturation of fat, the endometrioma remains hyperintense (d)

These sequences, which are based on the T1 relaxation time, yield a non-specific saturation, which may suppress nonfatty tissues with similar T1 values, such as the blood when methemoglobin is present, thus leading to a difficult differential diagnosis between mature cystic teratomas and endometriomas (Fig. 2) [34, 35]. Selective saturation of fatty tissue can be obtained with spectral saturation (SPAIR or SPIR) used in 2D SPIR T1 images or in 3D interpolated sequences, such as THRIVE or DIXON.

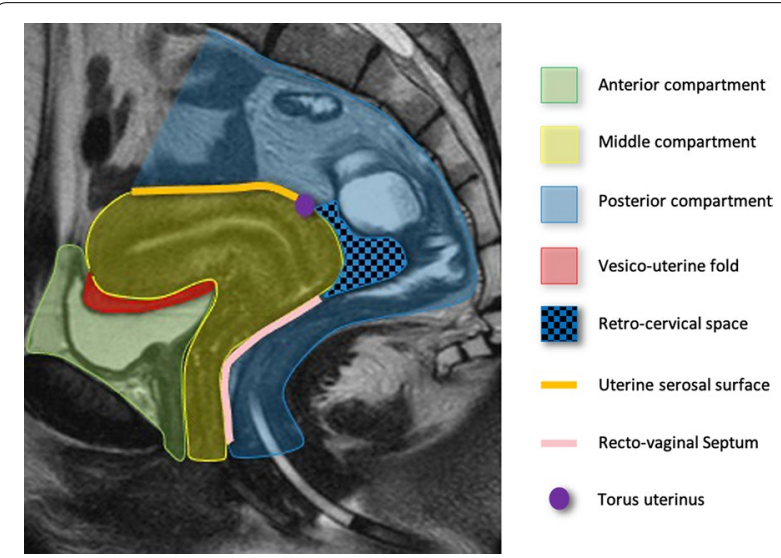

Fig. 3 Midsagittal T2w image of female pelvis with main anatomic landmarks to be considered in the evaluation of DIE

\section{MAYBES}

The use of intravenous contrast media is widely debated in the literature. Deep endometriosis is recognized by a low signal intensity tissue with small hyperintense foci in T2w images, which may also show distortion of the pelvic anatomy associated with adhesions. Therefore, contrastenhanced (CE) images appear to be useless in the diagnosis of DIE. However, for specific indications, the injection of Gd-based contrast agent may be advisable. CE-images are mandatory in cases of complex adnexal hemorrhagic cysts showing mural thickening or other potentially malignant features in T2w images. Similarly, the use of contrast agents may aid in differentiating endometriomas from luteal ovarian cysts or tubo-ovarian abscesses [36, 37]. In our center, we have found that combining MR colonography with CE THRIVE images may enable the diagnosis of colorectal involvement by less experienced radiologists thanks to an easier recognition of thickened wall and for the possibility to distinguish enhancing nodules from endoluminal fecal material or air [38]. Postcontrast MR urography should be used when the ureteral involvement is suspected to define the degree of urinary tract dilation and the precise site of infiltration [39].

No consensus exists in the literature regarding the usefulness of vaginal and rectal opacification for the diagnosis of DIE; some authors find them extremely useful and have proposed the use double contrast barium enema or cross-sectional colonography with either CT or MRI 

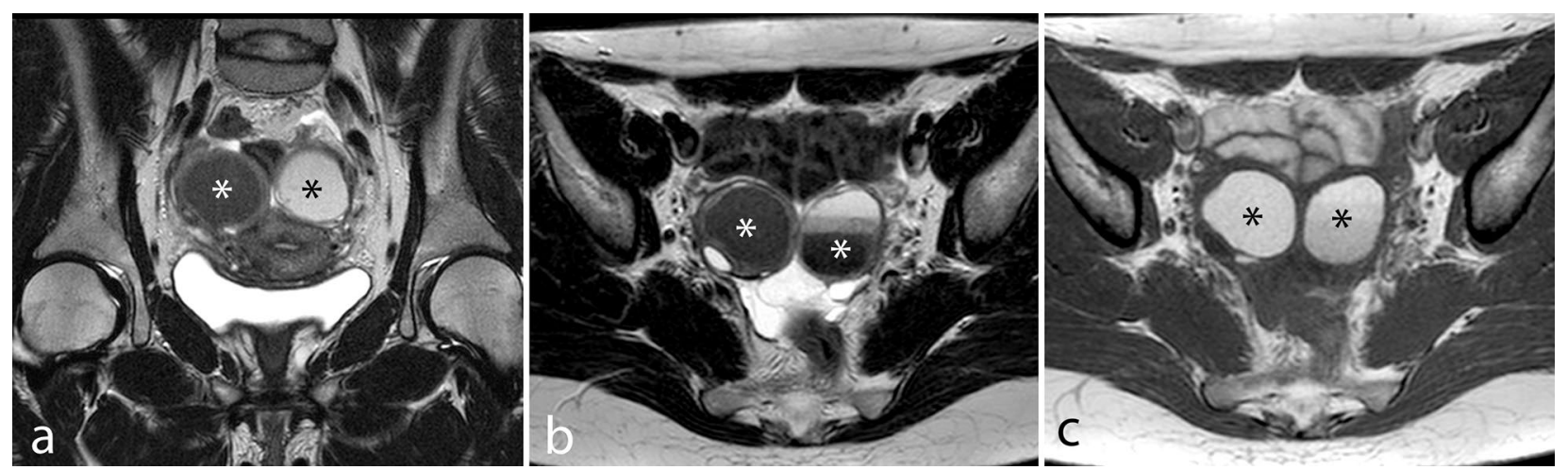

Fig. 4 Bilateral OMA. TSE T2 coronal (a) and axial (b) images and TSE T1w axial image (c). Bilateral endometriomas; the left-sided endometrioma shows a stratified aspect in b (shading sign). Of note, the ovaries are prolapsed in the pouch of Douglas, touching each other at the midline (kissing ovary sign)

[40-42], whereas others have reported no diagnostic improvement from these procedures [43]. In our center, we use rectal distension in patients showing an endometriotic lesion infiltrating the rectum in standard TSE T2w images to quantify the stenosis, which according to our experience is predictive of the need for bowel resection [25]. However, several alternative methods based on T2w images without rectal distension have been described to predict the need for bowel resection $[44,45]$.

\section{MR anatomic landmarks}

The imaging evaluation of endometriosis should be guided by the statistical frequency of involvement of the pelvic anatomy [11] and be consistently accurate.
To achieve a uniform evaluation of women with suspected endometriosis, the IDEA group in 2016 and the society of Abdominal Radiology have proposed a consensus lexicon for reporting US and MRI, respectively $[24,46]$. In both experiences, it is suggested to report findings by pelvic compartments (anterior, middle and posterior) and using consistent anatomic landmarks (Fig. 1, 3).

Anterior compartment is the space limited anteriorly by the pubic symphysis and posteriorly by the uterus and contains the urinary bladder, the vesico-uterine fold and the round ligaments. The middle compartment contains the uterus and the ovaries, while the posterior compartment can be divided into the recto-uterine,
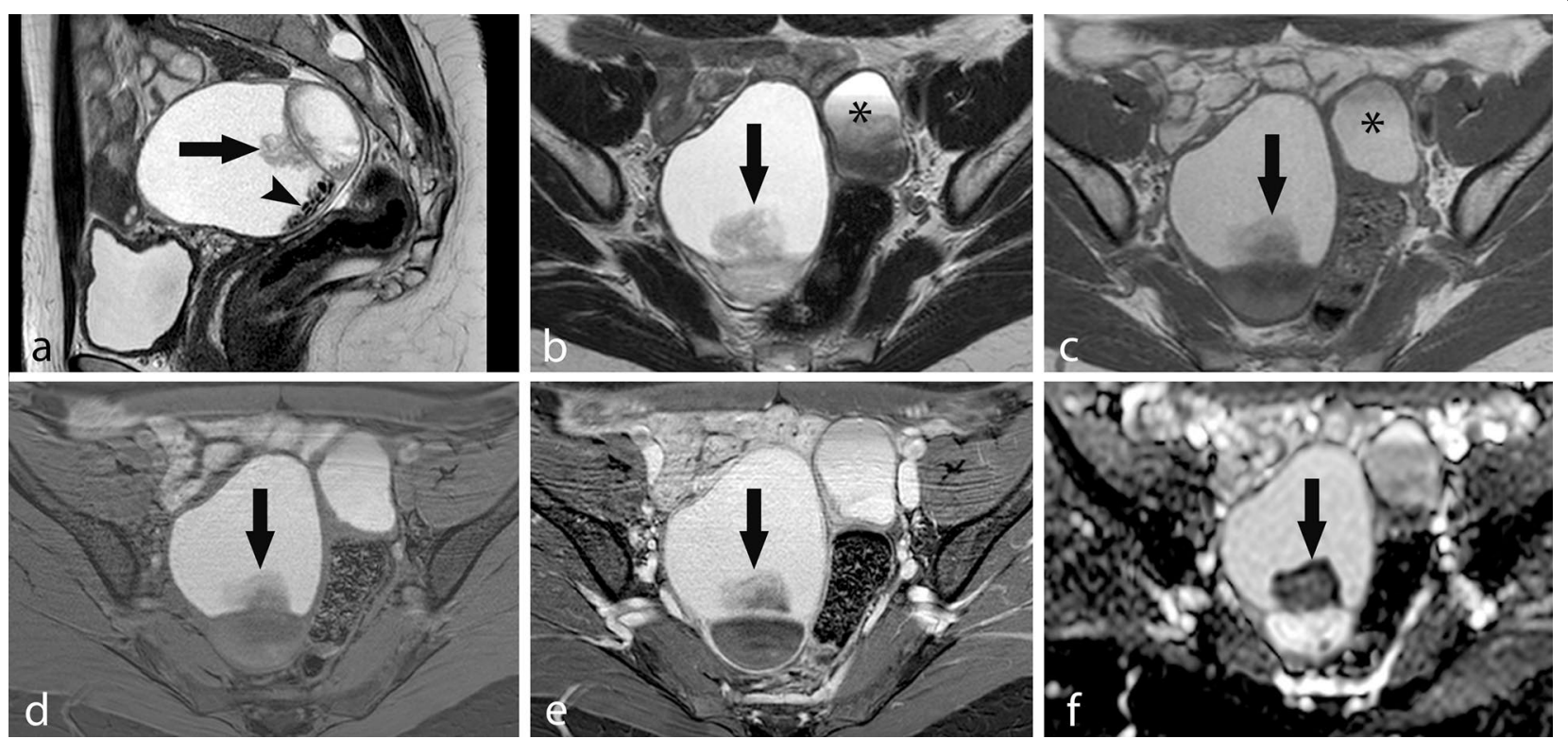

Fig. 5 Large OMA with irregular mural vegetation and dark spots. TSE T2w sagittal image (a), TSE T2w axial image (b), TSET1w axial image (c), axial THRIVE image (d), contrast-enhanced axial THRIVE image (e) and ADC map (f). A large right multiloculated OMA is shown with an irregular mural vegetation (arrow) and small dark spots (arrowhead). The vegetation shows no significant contrast enhancement (e) but restricted diffusion (f) and should be considered suspected for malignancy. A smaller left-sided OMA with shading sign is also visible $\left(^{*}\right)$ 

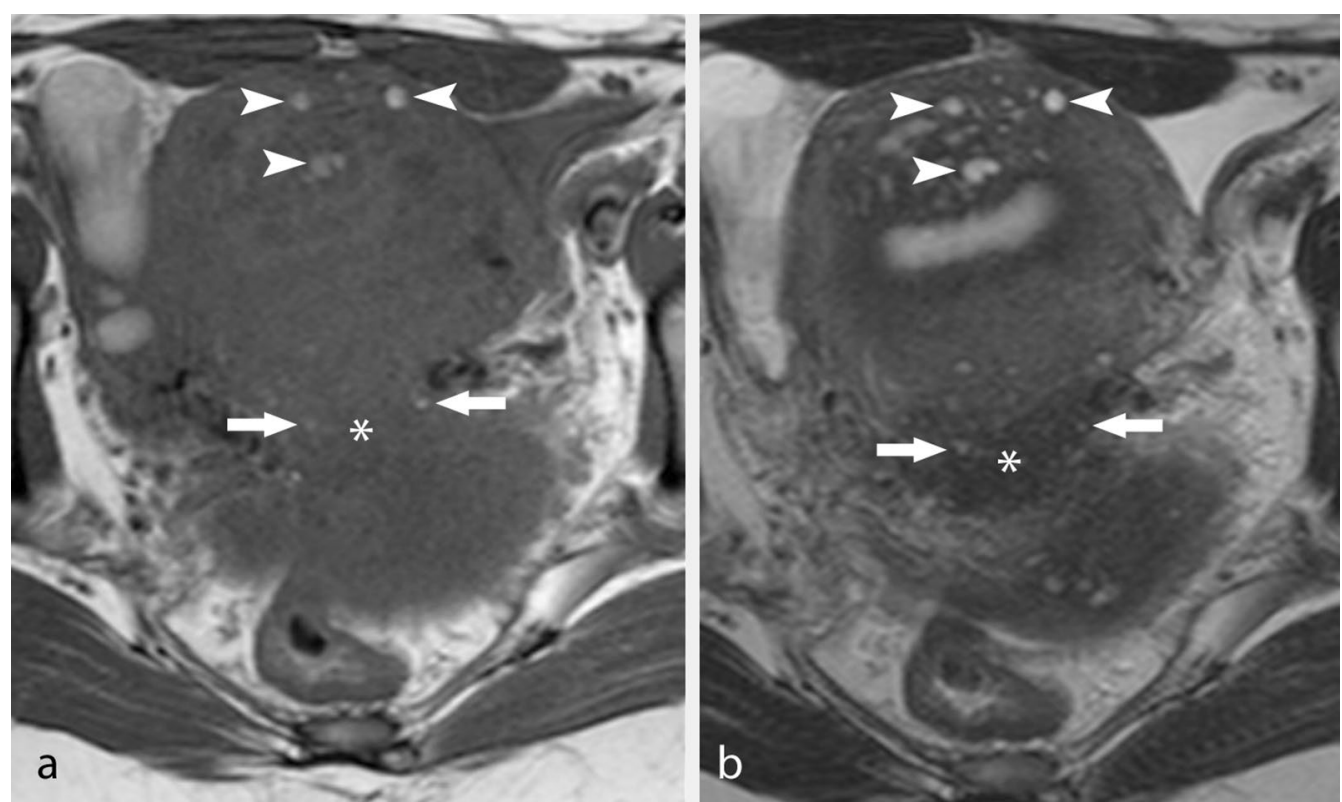

Fig. 6 Retro-uterine DIE nodule. TSET1w image and TSE T2w image. DIE nodules are characterized by intermediate signal intensity in T1w images $\left(^{*}\right.$ in $\mathbf{a}$ ) and low signal intensity in T2w images (* in $\mathbf{b}$ ), with high intensity foci in both sequences (arrows). Adenomyosis is also shown with a similar MR aspect within the anterior wall of the uterus (arrowheads)

recto-cervical spaces and the recto-vaginal septum and contains the serosal surface of the uterus, the pouch of Douglas, the torus uterinus, the USL well as the rectum and the sigmoid colon.

\section{MRI findings}

Endometriosis is a multifocal disease that may involve multiple pelvic structures with possible extra-pelvic extension. OMA, superficial peritoneal lesions, and DIE have been reported in surgical series studies to affect the ovaries in $65-80 \%, 45-50 \%$, and $63-70 \%$ of women, respectively [12, 47, 48]. DIE is usually more frequent in the posterior pelvic compartment (95\% of cases) including the torus uterinus, the recto-vaginal septum, USL, pouch of Douglas and anterior wall of the rectum than the anterior pelvic compartment (including the

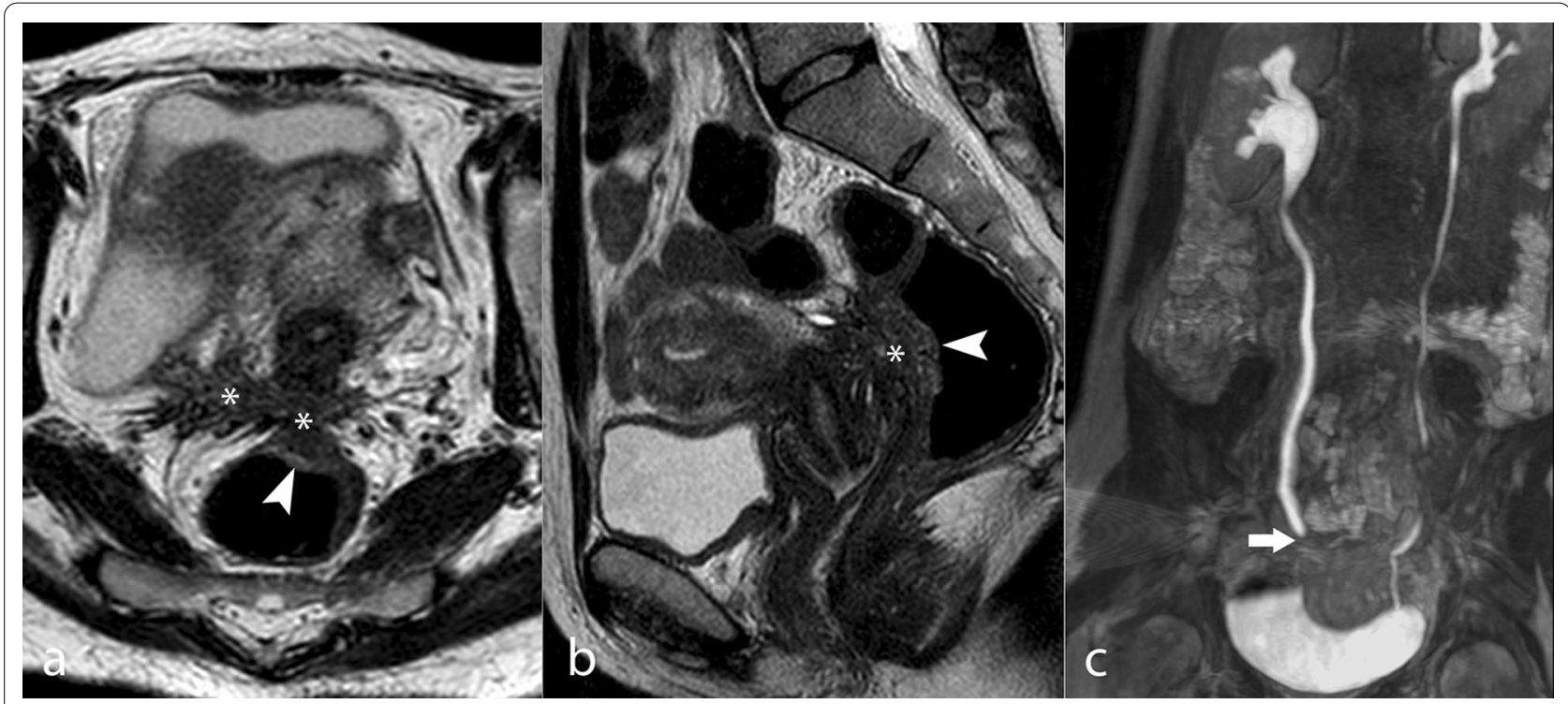

Fig. 7 Severe DIE with ureteral involvement. TSE T2w axial image (a), TSET2w sagittal image (b) and CE MR urography (c). Large DIE nodule of the pouch of Douglas extending to the right USL $\left(^{*}\right)$ and the anterior rectal wall (arrowhead). MR urography shows the involvement of the right pelvic ureter (arrow), with consequent moderate hydronephrosis 

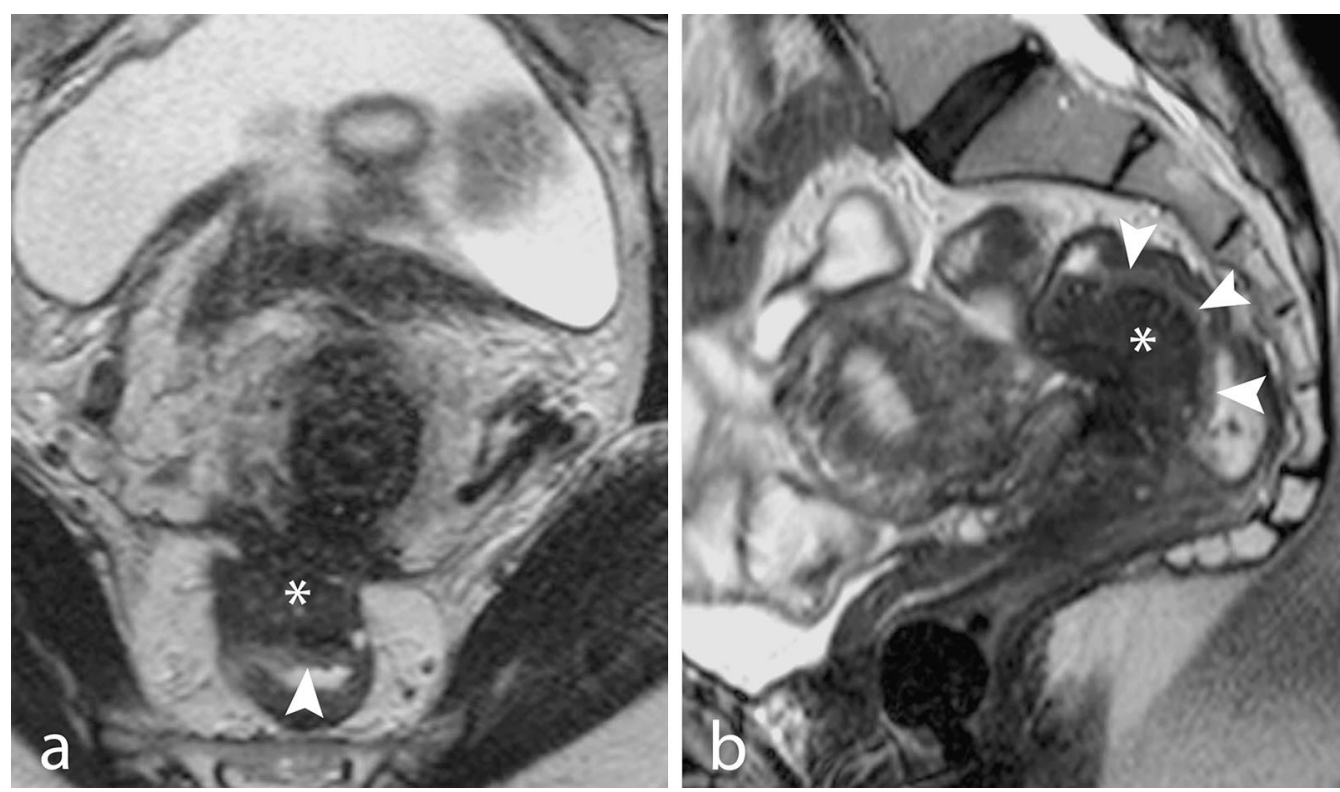

Fig. 8 DIE with rectal infiltration. TSET2w axial $(\mathbf{a})$ and sagittal $(\mathbf{b})$ images. A large DIE nodule $\left(^{*}\right)$ infiltrates the anterior wall of the rectum. The nodule has a mushroom-cap shape with a bright peripheral rim (arrowhead) corresponding to a normal mucosa layer
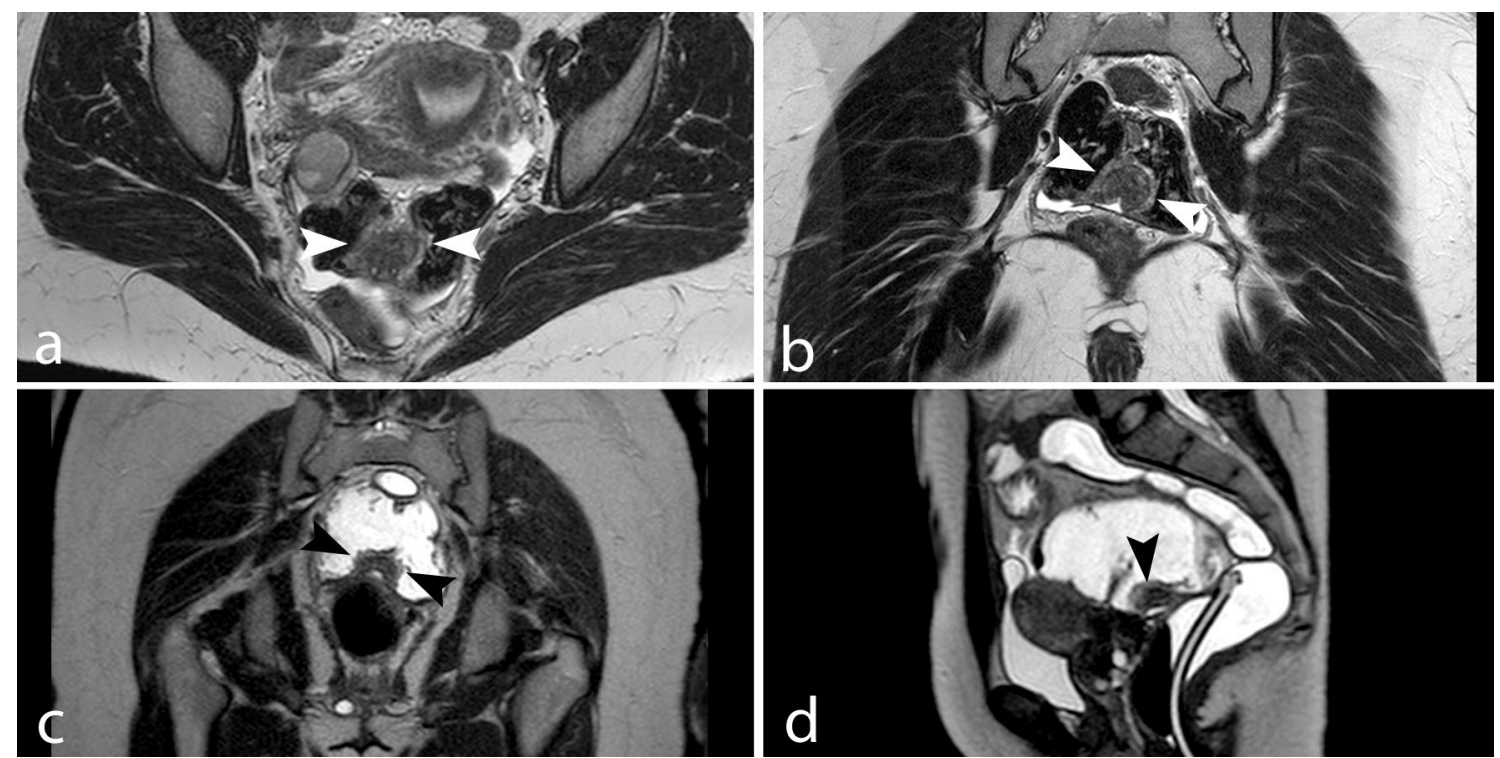

Fig. 9 DIE with cecal infiltration. TSE T2w axial (a) and coronal (b) images, SSFSET2 MR-colonography coronal (c) and sagittal (d) images. A large DIE nodule with a mushroom shape (arrowheads) infiltrates the cecum which has a pelvic position in the pouch of Douglas

vesico-uterine pouch and bladder; $16 \%$ of cases). Both compartments may be involved in approximately $10 \%$ of cases, whereas ureter and nerve lesions are seen in $5 \%$ of patients $[47,49]$.

OMA may manifest as solitary or multiple thick-walled cysts showing homogeneous high signal intensity in $\mathrm{T} 1 \mathrm{w}$ and fat-saturated T1w images regardless of the intensity in $\mathrm{T} 2 \mathrm{w}$ images. According to the age at bleeding onset, OMA may be either hyperintense or hypointense in T2w images or may show a typical stratified appearance (shading sign), as a result of cyclic bleeding with blood products accumulating over the course of months (Fig. 4) [37]. 


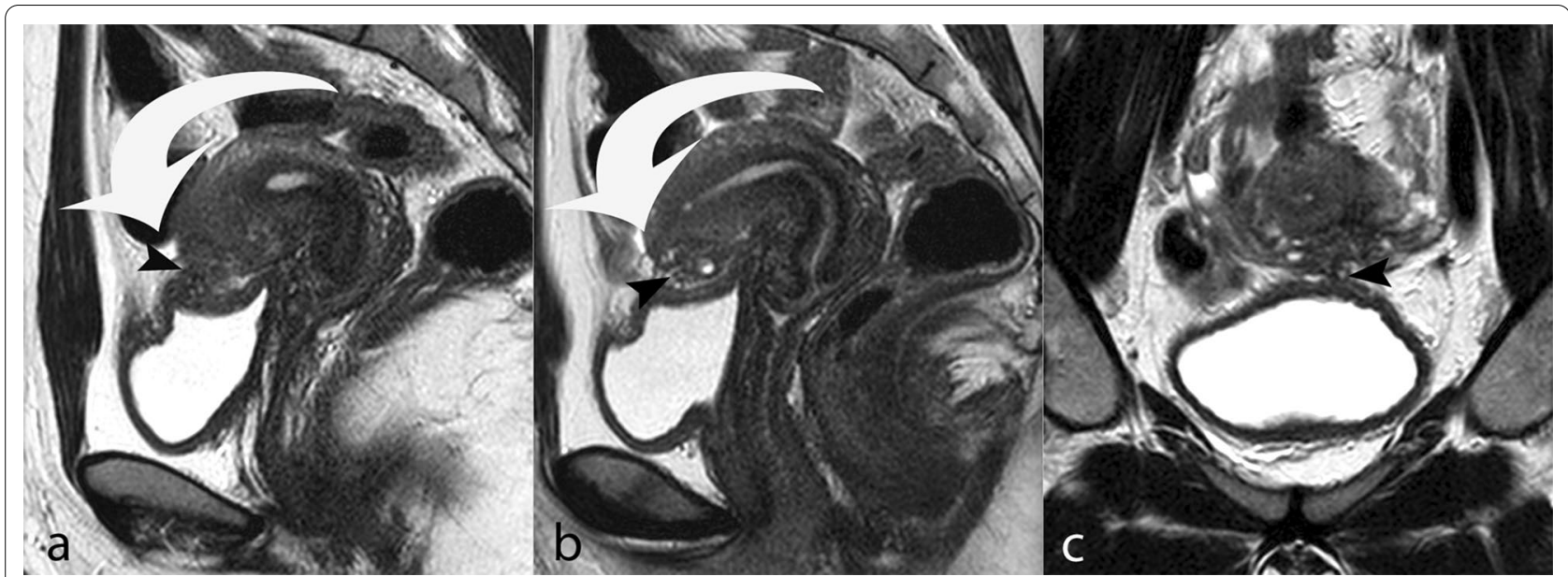

Fig. 10 DIE with vesical infiltration. TSE T2w sagittal $(\mathbf{a}, \mathbf{b})$ and coronal (c) images. The vesico-uterine fold is occupied by a DIE nodule (arrowhead) anteriorly tethering the uterine body (curved arrow)
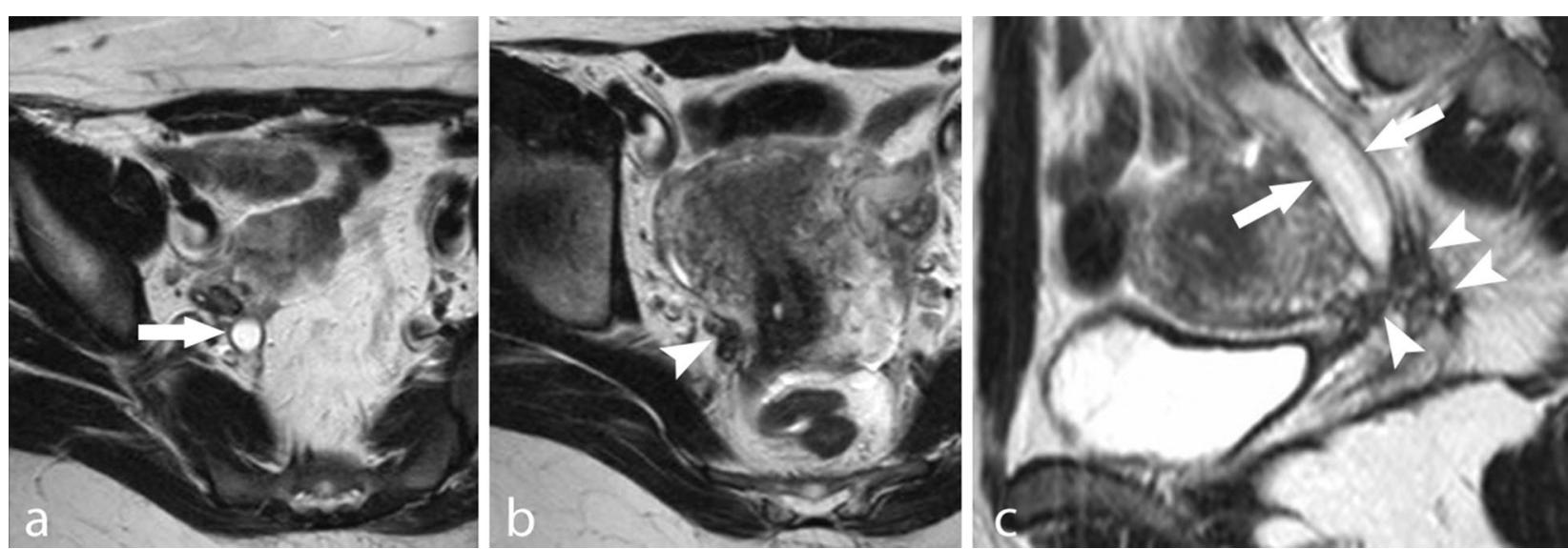

Fig. 11 DIE with right ureter infiltration. TSE T2w axial $(\mathbf{a}, \mathbf{b})$ and sagittal (c) images. A right para-uterine DIE nodule (arrowheads) infiltrates the distal tract of the right ureter, which is dilated (arrows)

In some cases, dark spots (low-intensity, well-defined images in T2w sequences) may be visible within cysts (Fig. 5) [50]. Irregular mural thickenings or mural vegetations should be studied after the intravenous injection of contrast agents and DWI sequences to exclude malignant transformation (Fig. 5).

DIE can manifest as pelvic nodules or plaque-like lesions and adhesions [51, 52]. Nodules and plaque-like lesions are composed of endometrial glands and stroma surrounded by a thick fibro-muscular and inflammatory reaction, and usually have an irregular, spiculated shape and a signal intensity similar to that of pelvic muscles, with intermediate signal intensity in T1w sequences and low signal intensity in T2w images. Small hyperintense foci corresponding to endometrial glands are almost always recognized within the endometriotic nodules in both T1w and T2w images (Fig. 6). The most common site of DIE nodules is the posterior pelvic compartment, where all anatomic structures bordering the pouch of Douglas can be involved (the posterior border of the cervix, the torus uterinus, the uterosacral ligaments, the vaginal wall, the anterior wall of the rectum and the recto-sigmoid junction; Fig. 7a, b) [53].

Diagnosis of bowel involvement is based on the presence of a nodular or plaque-like endometriotic bowel wall thickening and loss of the fat tissue plane between the intestinal loop and the uterus or other adjacent organs. The most frequent sites of bowel endometriosis 


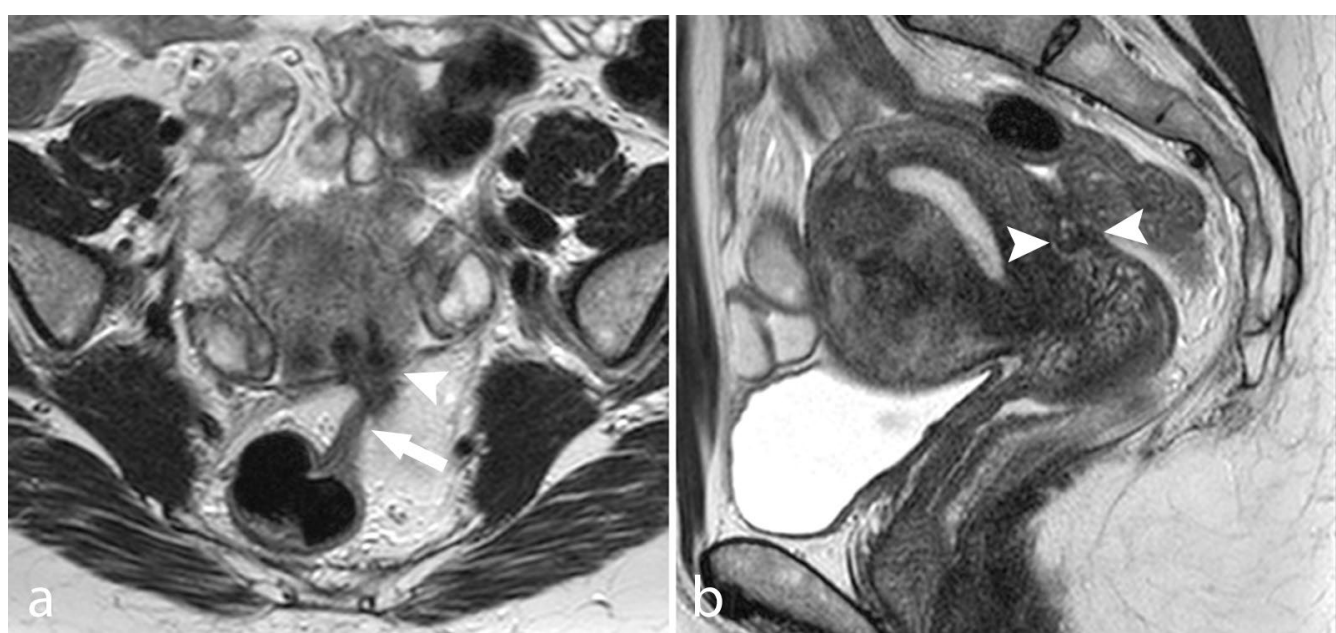

Fig. 12 Adhesive obliteration of the pouch of Douglas. TSE T2w axial (a) and sagittal (b) images. The anterior rectal wall is tethered (arrow) to the posterior surface of the uterus, where a DIE nodule is seen (arrowhead)

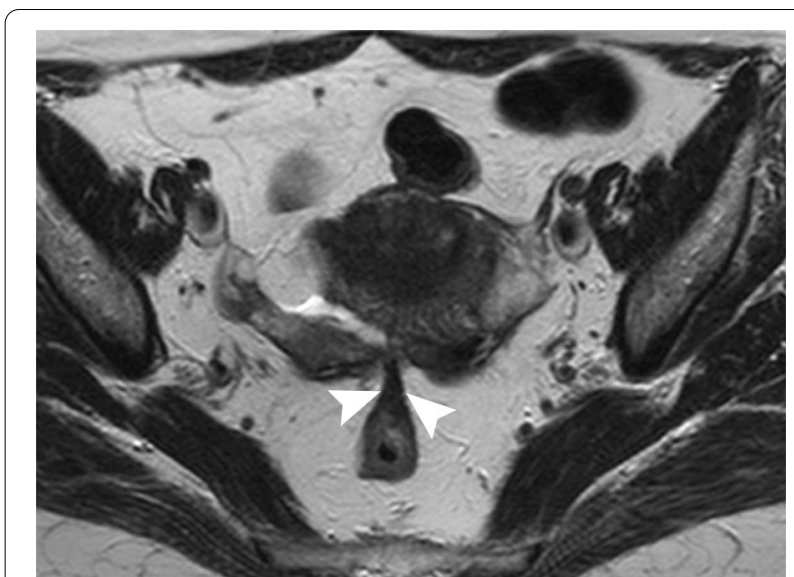

Fig. 13 DIE of the pouch of Douglas. TSE T2w axial image. Teardrop deformation of the rectum for DIE adhesions (arrowheads) is seen are the rectum and the sigmoid colon, while the involvement of the cecum or the ileum can be found in about $5 \%$ cases (Table 1). The diagnosis may be facilitated by the presence of ancillary findings such as a "mushroom cap" sign (Figs. 8, 9) [54]. This sign can be visible in any of the plane of the space and represents the endometriotic nodule growing into a mushroom-like shape in the bowel wall, covered by a high intensity signal rim representing the normal mucosa and submucosal layer (Figs. 7, 8, 9).

Endometriotic nodules of the anterior or lateral pelvic compartment are less frequently observed and usually involve the urinary system, particularly the vesical dome for nodules of the vesico-uterine fold (Fig. 10) and the ureters for lesions extending in the para-vesical space (Fig. 11). Axial and sagittal TSE T2w images are the most sensitive in identifying ureteral nodules; however,

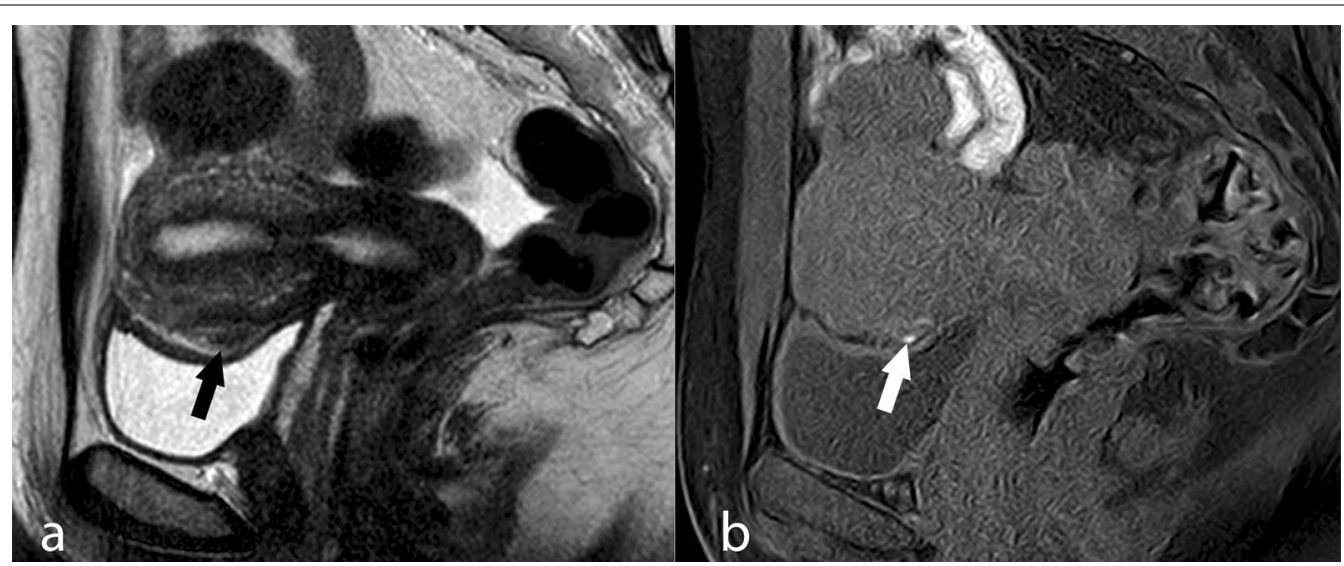

Fig. 14 Adhesive endometriosis of the vesico-uterine pouch. TSE T2w (a) and THRIVE (b) sagittal image. Small hyperintense spot-like images of the vesico-uterine pouch are seen. An endometriotic plaque was found through laparoscopy 

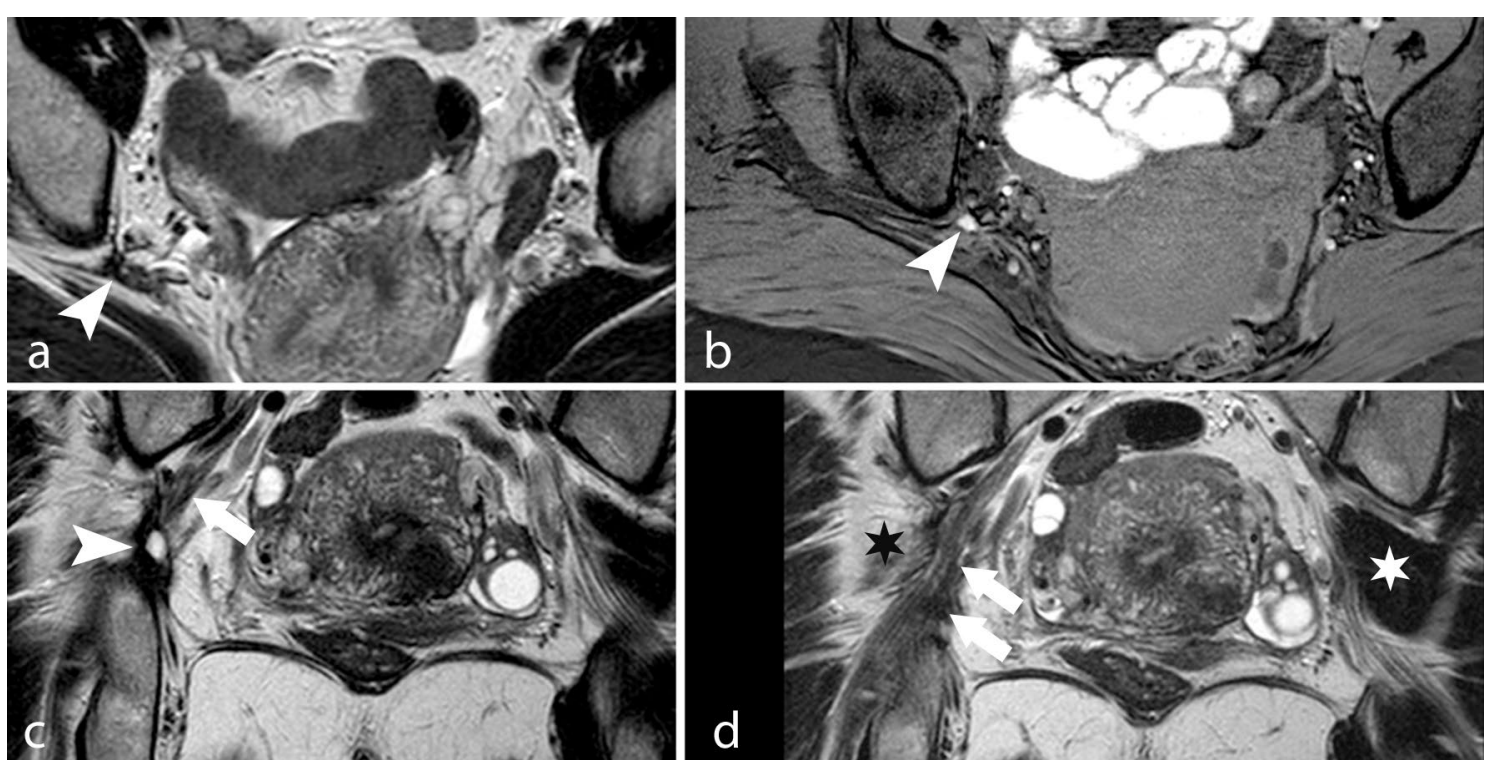

Fig. 15 Neural endometriosis. TSE T2w axial image (a), THRIVE axial image (b) and TSE T2w coronal images (c, d). A DIE nodule of the ischiatic foramen (arrowhead) surrounds the ischiatic nerve (arrows). Note the atrophy of the right piriformis muscle (black star) and normal left piriformis muscle (white star)

in these cases, the examination should be completed with post-contrast MR urography to demonstrate even mild urinary dilatation and the exact position of ureteral involvement (Fig. 7c).

In many cases, MRI may depict pelvic changes consistent with the presence of adhesions, which indirectly suggest DIE. In general, adhesions are suspected when fatty interfaces between adjacent structures are not clearly visible in any orthogonal planes. The most reliable finding to diagnose endometriotic adhesions is tethering and angulation of normal pelvic structures and bowel loops (Fig. 12). Adhesion between the anterior wall of the rectum and the posterior surface of the uterus, with a consequent "teardrop" deformation of the rectum and retroversion of the uterine body, is frequently seen in pelvic MRI and is specific for DIE (Fig. 13). Similarly, ovaries may prolapse in the Douglas pouch and create adhesions between each other and the uterine wall on the midline, thus producing a so-called kissing ovary sign, which is a common finding in DIE of the posterior pelvis (Fig. 4). Douglas obliteration should be suspected when nodules extend from the retro-cervical space to the anterior wall of the rectum or when adhesions are seen at this level (Figs. 7, 8, 12). In contrast, if small bowel loops are seen between the uterus and the rectum, the obliteration of the pouch of Douglas can be ruled out [53].

Anterior pelvic adhesions usually occur between the uterus and the bladder for plaque-like or linear implants in the vesico-uterine pouch, which may be visible as small spots with high signal intensity in the sagittal T1w fatsaturated images (Fig. 14) but can nonetheless be easily missed by pelvic MRI, whereas endo-vaginal US, owing to its ability to show an absence of sliding of the uterus along the bladder surface, is by far more sensitive.

Neural endometriosis is a rare condition characterized by perimenstrual radicular pain with no evidence of any alteration of the lumbar spine. The most affected nerves are the sacral plexus (57\% of cases) and the sciatic nerve (39\% of cases) $[49,55]$. MRI is the method of choice for the diagnosis of neural endometriosis, because transvaginal ultrasound cannot depict this anatomic area. The diagnosis relies on the recognition of endometriotic nodules along pelvic nerves and on indirect signs such as denervation muscular atrophy of the affected site (Fig. 15).

Adenomyosis in $30 \%$ of cases is associated with DIE; the presence of ill-defined nodules with hyperintense foci within uterine wall in T1w and T2w images or a thickening of the junctional zone $>12 \mathrm{~mm}$, is the most common findings of this specific condition (Fig. 6); however, the description of adenomyosis is beyond the purpose of this paper and is detailed elsewhere $[27,56]$.

\section{Conclusion}

Because of its extreme clinical heterogeneity, pelvic endometriosis remains challenging to diagnose. Current evidence demonstrates that the disease should be diagnosed non-invasively by combining the 
information from patient history, clinical examination, imaging and response to medical treatment [7]. Because the diagnostic accuracy of MRI may differ depending on radiologist experience, this review article aims to help radiologists obtain meaningful images with a tailored MR-acquisition protocol and recognize a wide range of pelvic changes that may result from endometriosis.

\begin{abstract}
Abbreviations
BTFE: Balanced turbo field echo; CE: Contrast enhanced; CT: Computed tomography; DIE: Deep pelvic infiltrating endometriosis; ESUR: European society of urogenital radiology; MRI: Magnetic resonance imaging; NE: Neural endometriosis; OMA: Ovarian endometrioma; RVS: Recto-vaginal septum; SSFSE: Single shot fast spin echo; SUP: Superficial peritoneal lesions; THRIVE: T1 high-resolution volume; TSE: Turbo spin echo; TVUS: Transvaginal ultrasound; USL: Utero-sacral ligaments.
\end{abstract}

\section{Authors' contributions}

FL, AS, MS contributed to conceptualization and drafting. NL, CL, AASI helped in drafting and revising. DS, DR, MDC, AS helped in image collection. All authors read and approved the final manuscript.

\section{Funding}

Not applicable.

\section{Availability of data and materials}

The datasets used and/or analyses during the current study are available from the corresponding author on reasonable request.

\section{Declarations}

Ethics approval and consent to participate Not applicable.

\section{Consent for publication}

Not applicable.

\section{Competing interests}

The authors declare that they have no competing interests.

\section{Author details}

${ }^{1}$ University of Bari Medical School - Interdisciplinary Department of Medicine, Section of Diagnostic Imaging, Piazza Giulio Cesare, 11, 70124 Bari, Italy. ${ }^{2}$ Unit of Gynecology, Mater Dei Hospital, Bari, Italy. ${ }^{3}$ Unit of Gynecology and Obstetrics, Di Venere Hospital, Bari, Italy. ${ }^{4}$ University of Bari Medical School - Interdisciplinary Department of Medicine, Section of Radiation Therapy, Bari, Italy.

Received: 23 May 2021 Accepted: 5 July 2021

Published online: 22 July 2021

\section{References}

1. Stepniewska A, Pomini P, Scioscia M, Mereu L, Ruffo G, Minelli L (2010) Fertility and clinical outcome after bowel resection in infertile women with endometriosis. Reprod Biomed Online 20:602-609

2. Bulun SE (2009) Endometriosis. N Engl J Med 360:268-279

3. Giudice LC, Kao LC (2004) Endometriosis. Lancet 364:1789-1799

4. Bazot M, Lafont C, Rouzier R, Roseau G, Thomassin-Naggara I, Darai E (2009) Diagnostic accuracy of physical examination, transvaginal sonography, rectal endoscopic sonography, and magnetic resonance imaging to diagnose deep infiltrating endometriosis. Fertil Steril 92:1825-1833

5. Noventa M, Scioscia M, Schincariol M et al (2019) Imaging modalities for diagnosis of deep pelvic endometriosis: comparison between trans-vaginal Sonography, rectal endoscopy Sonography and magnetic resonance imaging. A head-to-head meta-analysis. Diagnostics (Basel) 9:225

6. Zondervan KT, Becker CM, Missmer SA (2020) Endometriosis. N Engl J Med 382:1244-1256

7. Chapron C, Marcellin L, Borghese B, Santulli P (2019) Rethinking mechanisms, diagnosis and management of endometriosis. Nat Rev Endocrinol 15:666-682

8. Agarwal SK, Chapron C, Giudice LC et al (2019) Clinical diagnosis of endometriosis: a call to action. Am J Obstet Gynecol 220:354 e351-354 e312

9. Scardapane A, Lorusso F, Scioscia M, Ferrante A, Stabile lanora AA, Angelelli G (2014) Standard high-resolution pelvic MRI vs. low-resolution pelvic MRI in the evaluation of deep infiltrating endometriosis. Eur Radiol 24:2590-2596

10. Stabile lanora AA, Moschetta M, Lorusso F et al (2013) Rectosigmoid endometriosis: comparison between CT water enema and video laparoscopy. Clin Radiol 68:895-901

11. Chamie LP, Blasbalg R, Pereira RM, Warmbrand G, Serafini PC (2011) Findings of pelvic endometriosis at transvaginal US, MR imaging, and laparoscopy. Radiographics 31:E77-100

12. Scardapane A, Lorusso F, Bettocchi S et al (2013) Deep pelvic endometriosis: accuracy of pelvic MRI completed by MR colonography. Radiol Med 118:323-338

13. Athey PA, Diment DD (1989) The spectrum of sonographic findings in endometriomas. J Ultrasound Med 8:487-491

14. Carbognin G, Guarise A, Minelli L et al (2004) Pelvic endometriosis: US and MRI features. Abdom Imaging 29:609-618

15. Koninckx PR, Meuleman C, Demeyere S, Lesaffre E, Cornillie FJ (1991) Suggestive evidence that pelvic endometriosis is a progressive disease, whereas deeply infiltrating endometriosis is associated with pelvic pain. Fertil Steril 55:759-765

16. Laux-Biehlmann A, d'Hooghe T, Zollner TM (2015) Menstruation pulls the trigger for inflammation and pain in endometriosis. Trends Pharmacol Sci 36:270-276

17. Vercellini P, Chapron C, Fedele L, Frontino G, Zaina B, Crosignani PG (2003) Evidence for asymmetric distribution of sciatic nerve endometriosis. Obstet Gynecol 102:383-387

18. Chapron C, Fauconnier A, Vieira M et al (2003) Anatomical distribution of deeply infiltrating endometriosis: surgical implications and proposition for a classification. Hum Reprod 18:157-161

19. Laufer MR, Goitein L, Bush M, Cramer DW, Emans SJ (1997) Prevalence of endometriosis in adolescent girls with chronic pelvic pain not responding to conventional therapy. J Pediatr Adolesc Gynecol 10:199-202

20. Schliep KC, Mumford SL, Peterson CM et al (2015) Pain typology and incident endometriosis. Hum Reprod 30:2427-2438

21. Dunselman GA, Vermeulen N, Becker C et al (2014) ESHRE guideline: management of women with endometriosis. Hum Reprod 29:400-412

22. Goncalves MO, Siufi Neto J, Andres MP, Siufi D, de Mattos LA, Abrao MS (2021) Systematic evaluation of endometriosis by transvaginal ultrasound can accurately replace diagnostic laparoscopy, mainly for deep and ovarian endometriosis. Hum Reprod 36:1492-1500

23. Guerriero S, Saba L, Pascual MA et al (2018) Transvaginal ultrasound vs magnetic resonance imaging for diagnosing deep infiltrating endometriosis: systematic review and meta-analysis. Ultrasound Obstet Gynecol 51:586-595

24. Guerriero S, Condous G, van den Bosch T et al (2016) Systematic approach to sonographic evaluation of the pelvis in women with suspected endometriosis, including terms, definitions and measurements: a consensus opinion from the International Deep Endometriosis Analysis (IDEA) group. Ultrasound Obstet Gynecol 48:318-332

25. Scardapane A, Lorusso F, Francavilla M et al (2017) Magnetic resonance colonography may predict the need for bowel resection in colorectal endometriosis. Biomed Res Int 2017:5981217

26. Chiantera V, Petrillo M, Abesadze E et al (2018) Laparoscopic neuronavigation for deep lateral pelvic endometriosis: clinical and surgical implications. J Minim Invasive Gynecol 25:1217-1223

27. Bazot M, Darai E (2018) Role of transvaginal sonography and magnetic resonance imaging in the diagnosis of uterine adenomyosis. Fertil Steril 109:389-397 
28. Soliman AM, Fuldeore M, Snabes MC (2017) Factors associated with time to endometriosis diagnosis in the United States. J Womens Health (Larchmt) 26:788-797

29. Bazot M, Bharwani N, Huchon C et al (2017) European society of urogenital radiology (ESUR) guidelines: MR imaging of pelvic endometriosis. Eur Radiol 27:2765-2775

30. Bazot M, Gasner A, Ballester M, Darai E (2011) Value of thin-section oblique axial T2-weighted magnetic resonance images to assess uterosacral ligament endometriosis. Hum Reprod 26:346-353

31. Gutzeit A, Binkert CA, Koh DM et al (2012) Evaluation of the anti-peristaltic effect of glucagon and hyoscine on the small bowel: comparison of intravenous and intramuscular drug administration. Eur Radiol 22:1186-1194

32. Gollub MJ, Arya S, Beets-Tan RG et al (2018) Use of magnetic resonance imaging in rectal cancer patients: Society of Abdominal Radiology (SAR) rectal cancer disease-focused panel (DFP) recommendations 2017. Abdom Radiol (NY) 43:2893-2902

33. Brown G, Daniels IR, Richardson C, Revell P, Peppercorn D, Bourne M (2005) Techniques and trouble-shooting in high spatial resolution thin slice MRI for rectal cancer. Br J Radiol 78:245-251

34. Krinsky G, Rofsky NM, Weinreb JC (1996) Nonspecificity of short inversion time inversion recovery (STIR) as a technique of fat suppression: pitfalls in image interpretation. AJR Am J Roentgenol 166:523-526

35. Siegelman ES, Oliver ER (2012) MR imaging of endometriosis: ten imaging pearls. Radiographics 32:1675-1691

36. Outwater E, Schiebler ML, Owen RS, Schnall MD (1993) Characterization of hemorrhagic adnexal lesions with MR imaging: blinded reader study. Radiology 186:489-494

37. Dias JL, Veloso Gomes F, Lucas R, Cunha TM (2015) The shading sign: is it exclusive of endometriomas? Abdom Imaging 40:2566-2572

38. Scardapane A, Bettocchi S, Lorusso F et al (2011) Diagnosis of colorectal endometriosis: contribution of contrast enhanced MR-colonography. Eur Radiol 21:1553-1563

39. Bielen D, Tomassetti C, Van Schoubroeck D et al (2020) IDEAL study: magnetic resonance imaging for suspected deep endometriosis assessment prior to laparoscopy is as reliable as radiological imaging as a complement to transvaginal ultrasonography. Ultrasound Obstet Gynecol 56:255-266

40. Faccioli N, Foti G, Manfredi R et al (2010) Evaluation of colonic involvement in endometriosis: double-contrast barium enema vs. magnetic resonance imaging. Abdom Imaging 35:414-421

41. Biscaldi E, Ferrero S, Fulcheri E, Ragni N, Remorgida V, Rollandi GA (2007) Multislice CT enteroclysis in the diagnosis of bowel endometriosis. Eur Radiol 17:211-219

42. Kikuchi I, Kuwatsuru R, Yamazaki K, Kumakiri J, Aoki Y, Takeda S (2014) Evaluation of the usefulness of the MRI jelly method for diagnosing complete cul-de-sac obliteration. Biomed Res Int 2014:437962

43. Bazot M, Gasner A, Lafont C, Ballester M, Darai E (2011) Deep pelvic endometriosis: limited additional diagnostic value of postcontrast in comparison with conventional MR images. Eur J Radiol 80:e331-339
44. Rousset P, Buisson G, Lega JC et al (2021) Rectal endometriosis: predictive MRI signs for segmental bowel resection. Eur Radiol 31:884-894

45. Brusic A, Esler S, Churilov L et al (2019) Deep infiltrating endometriosis: Can magnetic resonance imaging anticipate the need for colorectal surgeon intervention? Eur J Radiol 121:108717

46. Jha P, Sakala M, Chamie LP et al (2020) Endometriosis MRI lexicon: consensus statement from the society of abdominal radiology endometriosis disease-focused panel. Abdom Radiol (NY) 45:1552-1568

47. Bazot M, Darai E, Hourani R et al (2004) Deep pelvic endometriosis: MR imaging for diagnosis and prediction of extension of disease. Radiology 232:379-389

48. Vimercati A, Achilarre MT, Scardapane A et al (2012) Accuracy of transvaginal sonography and contrast-enhanced magnetic resonance-colonography for the presurgical staging of deep infiltrating endometriosis. Ultrasound Obstet Gynecol 40:592-603

49. Siquara De Sousa AC, Capek S, Amrami KK, Spinner RJ (2015) Neural involvement in endometriosis: review of anatomic distribution and mechanisms. Clin Anat 28:1029-1038

50. Corwin MT, Gerscovich EO, Lamba R, Wilson M, McGahan JP (2014) Differentiation of ovarian endometriomas from hemorrhagic cysts at MR imaging: utility of the T2 dark spot sign. Radiology 271:126-132

51. Kataoka ML, Togashi K, Yamaoka T et al (2005) Posterior cul-de-sac obliteration associated with endometriosis: MR imaging evaluation. Radiology 234:815-823

52. Siegelman ES, Outwater E, Wang T, Mitchell DG (1994) Solid pelvic masses caused by endometriosis: MR imaging features. AJR Am J Roentgenol 163:357-361

53. Loubeyre $P$, Petignat $P$, Jacob S, Egger JF, Dubuisson JB, Wenger JM (2009) Anatomic distribution of posterior deeply infiltrating endometriosis on MRI after vaginal and rectal gel opacification. AJR Am J Roentgenol 192:1625-1631

54. Yoon JH, Choi D, Jang KT et al (2010) Deep rectosigmoid endometriosis: "mushroom cap" sign on T2-weighted MR imaging. Abdom Imaging 35:726-731

55. Ceccaroni M, Clarizia R, Cosma S, Pesci A, Pontrelli G, Minelli L (2011) Cyclic sciatica in a patient with deep monolateral endometriosis infiltrating the right sciatic nerve. J Spinal Disord Tech 24:474-478

56. Takeuchi M, Matsuzaki K (2011) Adenomyosis: usual and unusual imaging manifestations, pitfalls, and problem-solving MR imaging techniques. Radiographics 31:99-115

\section{Publisher's Note}

Springer Nature remains neutral with regard to jurisdictional claims in published maps and institutional affiliations.

\section{Submit your manuscript to a SpringerOpen ${ }^{\circ}$ journal and benefit from:}

- Convenient online submission

- Rigorous peer review

- Open access: articles freely available online

- High visibility within the field

- Retaining the copyright to your article

Submit your next manuscript at springeropen.com 